\title{
Lepton Flavor Violation and Bilinear R-parity Violation
}

\author{
Kiwoon $\mathrm{Choi}^{a, b}$, Eung Jin Chun ${ }^{c, d}$ and Kyuwan Hwang ${ }^{b}$ \\ ${ }^{a}$ Santa Cruz Institute for Particle Physics, Santa Cruz, CA 95064, U. S. A. \\ ${ }^{b}$ Korea Advanced Institute of Science and Technology, Taejeon 305-701, Korea \\ ${ }^{c}$ Korea Institute for Advanced Study, Seoul 130-012, Korea \\ ${ }^{d}$ Department of Physics, Lancaster University, Lancaster LA1 4YB, UK
}

\begin{abstract}
We examine some flavor changing processes such as rare leptonic decays of the long-lived neutral kaon, muon-electron conversion in nuclei and radiative muon decay which are induced by the combined effects of bilinear and trilinear R-parity violations. These processes are used to put strong constraints on certain products of the bilinear and trilinear couplings. We also discuss the constraints on R-parity violation from neutrino masses and compare them with the constraints from flavor changing decay processes. Large range of parameter space satisfying the constraints from neutrino masses can be excluded by the flavor changing processes considered in this paper, and also vice versa.
\end{abstract} PACS number(s): 11.30.Fs, 12.60.Jv, 13.35.Bv, 13.40.Hq, 14.60.Pq 
R-parity violation in the minimal supersymmetric standard model can be an interesting source of lepton and quark flavor violation. Typically one obtains bounds on R-parity violating couplings from the non-observation of such flavor violating phenomena in experiments. There are a vast of literatures addressing this issue [1]. However most of them have focused on the effects of trilinear R-parity violation alone. In generic context, both bilinear and trilinear R-parity violations exist and they are independent from each other. Our focus in this paper is on the effects of bilinear R-parity violation on some flavor changing processes such as $K_{L} \rightarrow e_{i} \bar{e}_{j}, K^{+} \rightarrow \pi^{+} \nu_{i} \bar{\nu}_{j}, \mu-e$ conversion in nuclei, and $\mu \rightarrow e \gamma$.

These processes can arise from tree or one-loop diagrams involving two insertions of the trilinear R-parity violating couplings $\lambda$ and $\lambda^{\prime}$. The resulting bounds on $\lambda \lambda$ and $\lambda^{\prime} \lambda^{\prime}$ are obtained in the literature for $K_{L} \rightarrow e_{i} \bar{e}_{j}$ [2], $K^{+} \rightarrow \pi^{+} \nu_{i} \bar{\nu}_{j}$ [3], $\mu-e$ conversion in nuclei 凷,5], and $\mu \rightarrow e \gamma$ [6]. Flavor changing decays of the neutral kaon and also the $\mu-e$ conversion in nuclei can be achieved by tree diagrams without any insertion of small fermion mass, thereby put very strong bounds of order $10^{-6} \sim 10^{-8}$ on $\lambda \lambda$ and $\lambda^{\prime} \lambda^{\prime}$. However one-loop diagrams for $\mu \rightarrow e \gamma$ need additional chirality flip by small fermion mass. The decay amplitude is then suppressed by $m_{\mu} / m_{\tilde{f}}$ with $m_{\tilde{f}}$ being a typical sfermion mass. This makes the bounds on $\lambda \lambda$ and $\lambda^{\prime} \lambda^{\prime}$ from $\mu \rightarrow e \gamma$ looser, which are of order $10^{-4}$.

In the presence of bilinear R-parity violation, there are additional contributions involving both bilinear and trilinear couplings. The importance of such contribution to fermion electric dipole moments have been discussed recently in Refs. [7,8]. In this case, the chirality structure of diagrams differs from the case with trilinear couplings only. For instance, there are one-loop diagrams for $\mu \rightarrow e \gamma$ without having the chirality flip by small fermion mass. As summarized in Table II, this leads to strong bounds of order $10^{-7}$ on $\epsilon \lambda$ where $\epsilon$ is a dimensionless parameter measuring the size of bilinear R-parity violation. (See Table II.) On the other hand, tree diagrams for neutral kaon decays have to come with another chirality flip by small fermion mass, thereby lead to looser bounds of order $10^{-4} \sim 10^{-5}$ on $\epsilon \lambda$ and $\epsilon \lambda^{\prime}$ as summarized in Table I.

Since R-parity violation is severely constrained by neutrino masses [9], it is quite tempting 
to compare the bounds of Tables I and II with those from neutrino masses [10 12]. The recent Super-Kamiokande [13] and other neutrino oscillation data can be well explained by assuming small Majorana neutrino masses: $m_{\nu} \sim 10^{-1} \mathrm{eV}$ or less. It has been argued that the existing neutrino data suggest that the maximal values of all $3 \times 3$ neutrino mass matrix elements do not exceed $1 \mathrm{eV}[10]$. Here we take this as a real bound on neutrino masses and derive the resulting constraints on R-parity violations which are summarized in Table III. As we will see, the constraints from neutrino masses and those of Tables I and II are complementary to each other. Large range of parameter space satisfying the conditions for small neutrino masses can be excluded by the bounds of Tables I and II, and also vice versa.

To proceed, let us first specify the parameter basis for our analysis. When R-parity conservation is not assumed, the MSSM allows for renormalizable lepton number (L) or baryon number (B) violating interactions. In this paper, we ignore B-violating couplings by simply assuming that they are small enough to ensure the proton stability. We will then work in the basis in which the standard Yukawa couplings of the quarks and leptons are diagonal and also the vacuum expectation values of sneutrinos vanish [14]. Since this choice is not invariant under the renormalization group evolution, it must be understood as a basis choice for the parameters renormalized at the weak scalef. In this basis, still the most generic R-parity violating superpotential is given by

$$
\Delta W=\epsilon_{i} \mu L_{i} H_{2}+\lambda_{i j k} L_{i} L_{j} E_{k}^{c}+\lambda_{i j k}^{\prime} L_{i} Q_{j} D_{k}^{c},
$$

where $H_{2}$ is the Higgs doublet superfield with the hypercharge $Y=1 / 2, L_{i}$ and $Q_{i}$ are the lepton and quark doublet superfields, $E_{i}^{c}$ and $U_{i}^{c}, D_{i}^{c}$ are the anti-lepton and anti-quark singlet superfields, respectively. Here the dimensionless parameters $\epsilon_{i}$ are introduced to

\footnotetext{
${ }^{1}$ The scale-dependence of choosing basis would motivate for a basis-independent prescription for R-parity violating physics as attempted in [15]. Making a basis-independent analysis for the processes under consideration is much more involved, so here we discuss everything in the specific basis with $\left\langle\tilde{\nu}_{i}\right\rangle=0$ applied for the parameters renormalized at the weak scale.
} 
parameterize the bilinear R-parity violation in unit of the supersymmetric Higgs mass parameter $\mu$. Bilinear R-parity violation can appear also in supersymmetry breaking scalar potential:

$$
\Delta V=B_{i} L_{i} H_{2}+m_{L_{i} H_{1}}^{2} L_{i} H_{1}^{\dagger}+\text { h.c. },
$$

where $H_{1}$ is the Higgs doublet with $Y=-1 / 2$. In our basis, $B_{i}$ and $m_{L_{i} H_{1}}^{2}$ are related by the condition of vanishing sneutrino vacuum value, $\left\langle\tilde{\nu}_{i}^{*}\right\rangle \propto B_{i}\left\langle H_{2}\right\rangle+m_{L_{i} H_{1}}^{2}\left\langle H_{1}\right\rangle=0$, so the entire bilinear R-parity violation are described by $\epsilon_{i}$ and $B_{i}$.

The basis described above is not the exact mass eigenbasis of fermions yet. If $\epsilon_{i} \neq 0$, there can be a mass-mixing of order $\epsilon_{i} \mu$ between R-parity even and R-parity odd fermions [16]. Before taking into account of this mixing, we have four types of fermion-scalar interaction vertices:

$$
g \phi^{*} \psi \tilde{G}, \quad h H \psi \psi, \quad h \phi \psi \tilde{H}, \quad \lambda \phi \psi \psi
$$

where the first three terms stand for R-parity conserving gauge, Yukawa and Higgsino vertices, respectively, and the last one does for R-parity violating Yukawa vertices. Here $\psi$ and $H$ collectively denote R-parity even matter fermions and Higgs scalars, respectively, and $\phi$, $\tilde{G}$ and $\tilde{H}$ are R-parity odd matter scalars, gauginos and Higgsinos, respectively. When written in terms of the mass eigenstate fermions, these couplings give rise to the mixing-induced vertices of the following types:

$$
\epsilon g \phi^{*} \psi \psi, \quad \epsilon h \phi \psi \psi, \quad \epsilon \lambda \phi \psi \tilde{G}, \quad \epsilon \lambda \phi \psi \tilde{H}
$$

where the first and second terms originate from the R-parity even gauge and Yukawa vertices in (3), respectively, while the other terms are from the R-parity odd Yukawa vertex in (3). Considering the processes connecting these mixing-induced vertices with those of (3), one can get bounds on the products of bilinear and trilinear couplings, which we will do explicitly in the subsequent discussion. There are also vertices induced by scalar mixing with $B_{i} \neq 0$ which we do not consider here. At this point, it is worthwhile to mention that the terms 
in (4) appear with $\left\langle\tilde{\nu}_{i}\right\rangle /\left\langle H_{1}\right\rangle$ instead of $\epsilon_{i}$, and with accordingly defined couplings $h$ and $\lambda$ in the different basis where the bilinear term $\epsilon_{i} \mu L_{i} H_{2}$ in the superpotential is rotated away. The most interesting feature of the mixing-induced vertices is that they have different chiral structure compared to their counterparts in (3) unless they do not include the R-parity conserving Yukawa coupling $h$, i.e. $\epsilon g \phi^{*} \psi \psi$ compared to $\lambda \phi \psi \psi$ and also $\epsilon \lambda \phi \psi \tilde{G}$ compared to $g \phi^{*} \psi \tilde{G}$. This allows for instance that, when combined with $g \phi^{*} \psi \tilde{G}$, the mixing-induced $\epsilon \lambda \phi \psi \tilde{G}$ generates a dipole moment of light fermion at one loop order without any insertion of the small Yukawa coupling of light fermion [7].

Let us write down explicitly the mixing-induced vertices which are relevant for our analysis. Ignoring the pieces involving the CKM matrix elements, the R-parity odd part is given by

$$
\begin{aligned}
& g \Theta_{i W}^{R *} e_{i}^{c}\left[d_{k} \tilde{u}_{k}^{*}+e_{k} \tilde{\nu}_{k}^{*}\right]+g \Theta_{i W}^{L *} e_{i}\left[u_{k} \tilde{d}_{k}^{*}+\nu_{k} \tilde{e}_{k}^{*}\right]+\sqrt{2} g\left[\Theta_{i B}^{N *} t_{W} Y_{f}+\Theta_{i W}^{N *} T_{f}^{3}\right] \nu_{i} f \tilde{f}^{*} \\
& -h_{i}^{e} \Theta_{j H}^{L *} e_{j}\left[e_{i}^{c} \tilde{\nu}_{i}+\nu_{i} \tilde{e}_{i}^{c}\right]-h_{i}^{d} \Theta_{j H}^{L *} e_{j}\left[d_{i}^{c} \tilde{u}_{i}+u_{i} \tilde{d}_{i}^{c}\right]+h_{i}^{u} \Theta_{j H}^{R *} e_{j}^{c}\left[u_{i}^{c} \tilde{d}_{i}+d_{i} \tilde{u}_{i}^{c}\right] \\
& +h_{i}^{e} \Theta_{j H_{1}}^{N *} \nu_{j}\left[e_{i}^{c} \tilde{e}_{i}+e_{i} \tilde{e}_{i}^{c}\right]+h_{i}^{d} \Theta_{j H_{1}}^{N *} \nu_{j}\left[d_{i}^{c} \tilde{d}_{i}+d_{i} \tilde{d}_{i}^{c}\right]-h_{i}^{u} \Theta_{j H_{2}}^{N *} \nu_{j}\left[u_{i}^{c} \tilde{u}_{i}+u_{i} \tilde{u}_{i}^{c}\right]
\end{aligned}
$$

where $l_{i}=\left(\nu_{i}, e_{i}\right)$ and $q_{i}=\left(u_{i}, d_{i}\right)$ are the lepton and quark doublets with the diagonal Yukawa couplings $h_{i}^{e}, h_{i}^{u}, h_{i}^{d}$ with the Higgs doublets, $e_{i}^{c}, u_{i}^{c}, d_{i}^{c}$ are the anti-lepton and anti-quark singlets, and the tilded fields stand for their scalar superpartners. Here $Y_{f}$ and $T_{f}^{3}$ denote the weak hypercharge and isospin of the fermion $f, \Theta_{i W, H}^{R}\left(\Theta_{i W, H}^{L}\right)$ are the mixing elements between the charged lepton $e_{i}^{c}\left(e_{i}\right)$ and the chargino $\tilde{W}^{+}, \tilde{H}^{+}\left(\tilde{W}^{-}, \tilde{H}^{-}\right)$, and $\Theta_{i B, W, H_{1}, H_{2}}^{N}$ is the mixing element between the neutrino $\nu_{i}$ and the neutralino $\tilde{B}, \tilde{W}_{3}, \tilde{H}_{1}, \tilde{H}_{2}$. The R-parity even part of the mixing-induced vertices which are relevant for our analysis is given by

$$
\Theta_{i \chi}^{N} \lambda_{i j k} \chi^{0}\left[e_{j} \tilde{e}_{k}^{c}+e_{k}^{c} \tilde{e}_{j}\right]+\Theta_{j W}^{L} \lambda_{i j k} \tilde{W}^{-}\left[\nu_{i} \tilde{e}_{k}^{c}+e_{k}^{c} \tilde{\nu}_{i}\right]+\Theta_{k W}^{R} \lambda_{i j k} \tilde{W}^{+}\left[\nu_{i} \tilde{e}_{j}+e_{j} \tilde{\nu}_{i}\right]
$$

where $\chi$ denotes the neutral gauginos $\tilde{B}$ and $\tilde{W}_{3}$. There are also similar vertices involving $\lambda_{i j k}^{\prime}$ which are not relevant for the discussion in this paper. The detailed form of the mixing elements $\Theta$ 's in Eqs.(5) and (6) can be found in Refs. [7, 12], however the following 
approximate expressions are enough for our purpose:

$$
\begin{aligned}
& \Theta_{i W}^{R} \approx \sqrt{2} \frac{m_{i}^{e}}{M_{1 / 2}} \frac{M_{W}}{M_{1 / 2}} \epsilon_{i} c_{\beta}, \quad \Theta_{i W}^{L} \approx \sqrt{2} \frac{M_{W}}{M_{1 / 2}} \epsilon_{i}^{*} c_{\beta} \\
& \Theta_{i B}^{N} \approx s_{W} \frac{M_{Z}}{M_{1 / 2}} \epsilon_{i}^{*} c_{\beta}, \quad \Theta_{i W}^{N} \approx-c_{W} \frac{M_{Z}}{M_{1 / 2}} \epsilon_{i}^{*} c_{\beta} \\
& \Theta_{i H}^{L} \approx 2 s_{\beta}\left(\frac{M_{W}}{M_{1 / 2}}\right)^{2} \epsilon_{i}^{*} c_{\beta}+\epsilon_{i}^{*}, \quad \Theta_{i H}^{R} \approx \frac{m_{i}^{e}}{c_{\beta} M_{1 / 2}} \epsilon_{i} c_{\beta} \\
& \Theta_{i H_{1}}^{N} \approx s_{\beta} \frac{M_{Z}^{2}}{\mu M_{1 / 2}} \epsilon_{i}^{*} c_{\beta}, \quad \Theta_{i H_{2}}^{N} \approx-c_{\beta} \frac{M_{Z}^{2}}{\mu M_{1 / 2}} \epsilon_{i}^{*} c_{\beta}
\end{aligned}
$$

where $m_{i}^{e}$ and $M_{1 / 2}$ denotes the charged lepton mass and the gaugino mass, respectively, $M_{W}$ and $M_{Z}$ are the weak gauge boson masses, $s_{W}=\sin \theta_{W}$ and $c_{W}=\cos \theta_{W}$ for the weak mixing angle $\theta_{W}, c_{\beta}=\cos \beta$ and $s_{\beta}=\sin \beta$ for $\tan \beta=\left\langle H_{2}\right\rangle /\left\langle H_{1}\right\rangle$.

Combined with trilinear R-parity violation, bilinear R-parity violation induces a left-right mixing of scalars which can contribute to the one-loop dipole moments of light fermions [8], and thus to $\mu \rightarrow e \gamma$. From the superpotential (1), one easily finds such type of mass mixing for squarks and sleptons:

$$
\left(\epsilon_{i}^{*} \lambda_{i j k} \mu v_{2}\right) \tilde{e}_{j} \tilde{e}_{k}^{c}+\left(\epsilon_{i}^{*} \lambda_{i j k}^{\prime} \mu v_{2}\right) \tilde{d}_{j} \tilde{d}_{k}^{c}+\text { h.c. },
$$

where $v_{2}=\left\langle H_{2}\right\rangle$. This left-right mixing gives rise to one-loop diagrams for $\mu \rightarrow e \gamma$ together with two insertions of the gauge vertices $g \phi^{*} \psi \tilde{G}$ without having any insertion of light fermion mass. We remark again that, in the basis where $L_{i} H_{2}$ in the superpotential is rotated away, the similar sfermion mixing terms arise from trilinear soft-terms with the coefficients $\left\langle\tilde{\nu}_{i}\right\rangle \lambda_{i j k}$ or $\left\langle\tilde{\nu}_{i}\right\rangle \lambda_{i j k}^{\prime}$.

We are now ready to consider the processes induced by diagrams connecting the mixinginduced vertices in (团) with the conventional vertices in (3). The processes $K_{L} \rightarrow e_{i} \bar{e}_{j}, K^{+} \rightarrow$ $\pi^{+} \nu_{i} \nu_{j}$ and also the $\mu-e$ conversion in nuclei can be triggered by tree diagrams involving both bilinear and trilinear R-parity violations. (See Fig. 1.) It is in fact straightforward to obtain the bounds on $\epsilon \lambda^{\prime}$ from these processes. The diagrams of Fig. 1 correspond to the diagrams which have been considered in Refs. [2-6] to obtain the bounds on $\lambda^{\prime} \lambda^{\prime}$, but here one $\lambda^{\prime}$ is replaced by appropriate mixing-induced coupling in (5) while properly taking into 
account the necessary chirality flip. The resulting bounds are summarized in Table I. Here the bounds on $\lambda_{i j k}^{\prime} \epsilon_{l} c_{\beta}$ are from diagrams with a mixing-induced vertex of the form $\epsilon g \phi^{*} \psi \psi$, while the bounds on $\lambda_{i j k}^{\prime} \epsilon_{l} / c_{\beta}$ are from those with $\epsilon h \phi \psi \psi$.

Let us now consider $\mu \rightarrow e \gamma$. As one can see in Fig. 2, the mixing-induced vertices of Eq.(6) can generate one-loop dipole moments of light fermions without having the insertion of light fermion mass. The required chirality flip is provided by the gaugino mass. Indeed, similar diagrams have been used to generate the leading contribution to the electric dipole moments of the electron and neutron in Ref. [7]. On the other hand, there are other type of one-loop diagrams for dipole moments without any light fermion mass. This type of diagrams involve the left-right sfermion mixing of Eq. (8) together with two insertions of the standard gaugino vertices. (See the diagram of Fig. 2b.) Note that there are two chirality flips in the internal lines of this type of diagram, one is the left-right sfermion mixing and the other is the gaugino mass mixing. For $\mu \rightarrow e \gamma$, there is only one diagram with $\tilde{B}-\tilde{W}_{3}$ mixing, giving somewhat smaller contributions.

The decay width of $\mu \rightarrow e \gamma$ is given by

$$
\Gamma(\mu \rightarrow e \gamma)=\frac{m_{\mu}^{3}}{16 \pi}\left(\left|\mathcal{B}_{L}\right|^{2}+\left|\mathcal{B}_{R}\right|^{2}\right)
$$

for the transition dipole moments

$$
\frac{i}{2} \bar{e} \sigma^{\mu \nu}\left(\mathcal{B}_{R} P_{R}+\mathcal{B}_{L} P_{L}\right) \mu F_{\mu \nu}
$$

where $P_{L, R}=\frac{1}{2}\left(1 \pm \gamma_{5}\right)$. The dominant contributions to $\mathcal{B}_{R, L}$ from the diagrams in Fig. 2 are found to be

$$
\begin{aligned}
\mathcal{B}_{R}=\frac{e g}{16 \pi^{2}}\{ & \frac{\Theta_{j W}^{L} \lambda_{1 j 2}}{m_{\tilde{\nu}_{1}}} G_{f}\left(m_{\tilde{W}} ; m_{\tilde{\nu}_{1}}\right) \\
& \quad-\frac{1}{\sqrt{2}} \frac{\lambda_{1 j 2}}{m_{\tilde{e}_{1}}}\left[\Theta_{j W}^{N} G_{s}\left(m_{\tilde{W}} ; m_{\tilde{e}_{1}}\right)+t_{W} \Theta_{j B}^{N} G_{s}\left(m_{\tilde{B}} ; m_{\tilde{e}_{1}}\right)\right] \\
& \left.+\sqrt{2} t_{W} \frac{\Theta_{j B}^{N} \lambda_{1 j 2}}{m_{\tilde{e}_{2}^{c}}} G_{s}\left(m_{\tilde{B}} ; m_{\tilde{e}_{2}^{c}}\right)\right\} \\
+ & \frac{e g^{2} t_{W}}{16 \pi^{2}} N_{B \chi_{n}} N_{W \chi_{n}} \frac{\epsilon_{j}^{*} \lambda_{j i k} \mu v_{2}}{m_{\tilde{e}_{i}} m_{\tilde{e}_{k}^{c}}} H_{s}\left(m_{\chi}^{0} ; m_{\tilde{e}_{i}}, m_{\tilde{e}_{k}^{c}}\right) \\
\mathcal{B}_{L}= & (1 \leftrightarrow 2) \quad \text { exchange of } \mathcal{B}_{R} .
\end{aligned}
$$


Here $N_{B \chi_{n}}$ and $N_{W \chi_{n}}$ are the diagonalization matrix bringing the neutralinos $\left(\tilde{B}, \tilde{W}_{3}, \tilde{H}_{1}^{0}, \tilde{H}_{2}^{0}\right)$ into the mass eigenstates $\chi_{n}$, and the loop functions $G_{f}, G_{s}$ and $H_{s}$ are given by

$$
\begin{aligned}
G_{f}\left(m_{f} ; m_{\alpha}\right) & =t\left[\frac{t^{2}-3}{2\left(1-t^{2}\right)^{2}}-\frac{2 \ln t}{\left(1-t^{2}\right)^{3}}\right], \\
G_{s}\left(m_{f} ; m_{\alpha}\right) & =t\left[\frac{t^{2}+1}{2\left(1-t^{2}\right)^{2}}+\frac{2 t^{2} \ln t}{\left(1-t^{2}\right)^{3}}\right], \\
H_{s}\left(m_{f} ; m_{\alpha}, m_{\beta}\right) & =\frac{m_{\alpha} m_{\beta}}{m_{\alpha}^{2}-m_{\beta}^{2}}\left[\frac{1}{m_{\alpha}} G_{s}\left(m_{f} ; m_{\alpha}\right)-\frac{1}{m_{\beta}} G_{s}\left(m_{f} ; m_{\beta}\right)\right],
\end{aligned}
$$

where $t=m_{f} / m_{\alpha}$.

Applying the experimental data [17] $\mathrm{BR}(\mu \rightarrow e \gamma)<4.9 \times 10^{-11}$ for the above transition dipole moment, one can derive bounds on the certain components of $\lambda_{i j k} \epsilon_{j}^{*}$ for reasonable range of superparticle spectrums. The results are summarized in Table II. In the absence of slepton generation mixing, $\mu \rightarrow e \gamma$ would provide bounds only on the components $\lambda_{1 j 2 \epsilon_{j}^{*}}$ and $\lambda_{2 j 1} \epsilon_{j}^{*}$. However the basis that we are working on is not the mass eigenbasis of sfermions, and thus there can be slepton generation mixing induced by the off-diagonal elements $\Delta_{i j}^{A B}$ of the slepton mass-squared matrix. (Here $A, B=L, R$ denote the chirality of the corresponding sleptons.) If we allow such slepton-mixing, other components of $\lambda_{i j k} \epsilon_{j}^{*}$ can be constrained also by $\mu \rightarrow e \gamma$ as in Table II.

Any lepton number violating interactions are highly constrained by neutrino masses. It is thus quite tempting to compare the constraints of Tables I and II with the constraints from neutrino masses. As mentioned before, we take the upper bound on each component of neutrino mass matrix to be $1 \mathrm{eV}$ [10], although it depends on the interpretation of SuperKamiokande data and also on the possible existence of light sterile neutrinos. Assuming a simple flavor structure of $\lambda_{i j k}$ and $\lambda_{i j k}^{\prime}$, strong bounds on $\lambda \lambda, \lambda^{\prime} \lambda^{\prime}$, and $\epsilon \epsilon$ were derived from neutrino oscillation data [11]. Here we briefly discuss this issue without any assumption on the flavor structure of $\lambda_{i j k}$ and $\lambda_{i j k}^{\prime}$, and compare the results with the constraints of Tables I and II. We will see that the constraints of Tables I and II from flavor changing processes are complementary to the constraints from neutrino masses. 
In the basis with vanishing sneutrino vacuum values, $\mathrm{R}$-parity violation are described by $\left\{\epsilon_{i}, B_{i}, \lambda_{i j k}, \lambda_{i j k}^{\prime}\right\}$. Here we are interested in the neutrino masses associated with $\epsilon_{i}, \lambda_{i j k}$ and $\lambda_{i j k}^{\prime}$, and thus ignore the contribution involving $B_{i}$. Then tree-diagrams with two insertions of the lepton-neutralino mixing give rise to

$$
\left(m_{\nu}\right)_{i j}^{\text {tree }} \sim \frac{M_{Z}^{2} c_{\beta}^{2}}{M_{1 / 2}} \epsilon_{i} \epsilon_{j},
$$

while one-loop diagrams with two insertions of $\lambda_{i j k}$ or of $\lambda_{i j k}^{\prime}$ give

$$
\begin{aligned}
&\left(\delta_{1} m_{\nu}\right)_{i j}^{\text {loop }} \sim \frac{3}{8 \pi^{2}} \frac{m_{k}^{d} m_{l}^{d}}{m_{\tilde{q}}^{2}} \lambda_{i k l}^{\prime} \lambda_{j l k}^{\prime}\left[A^{d *}+\mu t_{\beta}\right], \\
&\left(\delta_{2} m_{\nu}\right)_{i j}^{\text {loop }} \sim \frac{1}{8 \pi^{2}} \frac{m_{k}^{e} m_{l}^{e}}{m_{\tilde{l}}^{2}} \lambda_{i k l} \lambda_{j l k}\left[A^{e *}+\mu t_{\beta}\right],
\end{aligned}
$$

where $m_{i}^{d}$ and $m_{i}^{e}$ are the down-type quark and charged lepton masses, $A^{d}$ and $A^{e}$ are the soft $A$-parameter for the trilinear couplings $H_{1} Q D^{c}$ and $H_{1} L E^{c}, m_{\tilde{q}}$ and $m_{\tilde{l}}$ are the squark and slepton masses, and $c_{\beta}=\cos \beta$ and $t_{\beta}=\tan \beta$. There are also one-loop diagrams giving neutrino masses proportional to the products of bilinear and trilinear couplings ${ }^{2}$. Transparent description of such one-loop diagrams can be made by the use of mass insertion approximation [19]. The mass insertion by $\epsilon_{i}$ can appear either on the external fermion (neutralino/neutrino) line or on the internal chargino/charged lepton line. For each of these two types, there are diagrams in which one vertex is given by R-parity odd Yukawa coupling $\left(\lambda\right.$ or $\left.\lambda^{\prime}\right)$ and the other by R-parity even gauge or Yukawa $\left(h^{e}\right.$ or $\left.h^{d}\right)$ coupling. It turns out that the diagrams with $h^{e}$ or $h^{d}$ are suppressed by two more powers of $m_{i}^{e}$ or $m_{i}^{d}$ which come from the chirality flips in the internal lepton/slepton or quark/squark lines. These one-loop diagrams give the following contributions [19]:

$$
\begin{aligned}
\left(\delta_{3} m_{\nu}\right)_{i j}^{\text {loop }} \sim & \frac{3 g}{16 \pi^{2}} \frac{M_{Z}}{\sqrt{2} M_{1 / 2}} c_{\beta}\left(\epsilon_{i} \lambda_{j k k}^{\prime}+\epsilon_{j} \lambda_{i k k}^{\prime}\right) m_{k}^{d} \\
& +\frac{3}{16 \pi^{2}} \frac{M_{Z}^{2}}{\mu M_{1 / 2}} s_{\beta} c_{\beta}\left(\epsilon_{i} \lambda_{j k k}^{\prime}+\epsilon_{j} \lambda_{i k k}^{\prime}\right) \frac{h_{k}^{d}\left(m_{k}^{d}\right)^{2}}{m_{\tilde{q}}^{2}}\left[A^{d *}+\mu t_{\beta}\right]
\end{aligned}
$$

\footnotetext{
${ }^{2}$ Such one-loop mass has been noticed also in Ref. [18] within the different parameter basis with $\epsilon_{i}=0$ but $\left\langle\tilde{\nu}_{i}\right\rangle \neq 0$.
} 


$$
\begin{aligned}
\left(\delta_{4} m_{\nu}\right)_{i j}^{\text {loop } \sim} & \frac{g}{16 \pi^{2}} \frac{M_{Z}}{\sqrt{2} M_{1 / 2}} c_{\beta}\left(\epsilon_{i} \lambda_{j k k}+\epsilon_{j} \lambda_{i k k}\right) m_{k}^{e} \\
& +\frac{1}{16 \pi^{2}} \frac{M_{Z}^{2}}{\mu M_{1 / 2}} s_{\beta} c_{\beta}\left(\epsilon_{i} \lambda_{j k k}+\epsilon_{j} \lambda_{i k k}\right) \frac{h_{k}^{e}\left(m_{k}^{e}\right)^{2}}{m_{\tilde{l}}^{2}}\left[A^{e *}+\mu t_{\beta}\right] \\
& +\frac{1}{16 \pi^{2}}\left(\epsilon_{k} \lambda_{i j k}\right) \frac{\left(h_{i}^{e} m_{i}^{e}-h_{j}^{e} m_{j}^{e}\right) m_{k}^{e}}{m_{\tilde{l}}^{2}}\left[A^{e *}+\mu t_{\beta}\right]
\end{aligned}
$$

where $h_{i}^{d}$ and $h_{i}^{e}$ are the diagonalized quark and lepton Yukawa couplings generating the masses $m_{i}^{d}$ and $m_{i}^{e}$. Requiring that all of these contributions are smaller than $1 \mathrm{eV}$, we obtained upper bounds of several combinations of couplings, which are summarized in Table III. The $t_{\beta}$ dependence in Table III comes from the sfermion mixing mass term involving $\mu t_{\beta}$.

Note that the tree level neutrino mass (13) is not suppressed by small fermion mass, and thus leads to a strong bound of order $10^{-5} \sim 10^{-6}$ on $\epsilon_{i}$. Since all the loop-induced neutrino masses are suppressed by some powers of small fermion masses as well as the loop suppression factor, the light generation components of R-parity violating couplings are not severely constrained by neutrino masses.

We have found that the consideration of flavor changing decays of the neutral kaon and also of $\mu-e$ conversion in nuclei put bounds of order $10^{-4} \sim 10^{-5}$ on $\lambda_{i j k}^{\prime} \epsilon_{l} c_{\beta}^{ \pm 1}$ as summarized in Table I. Also $\mu \rightarrow e \gamma$ constrain $\lambda_{i 12} \epsilon_{i}^{*}$ and $\lambda_{i 21} \epsilon_{i}^{*}$ to be less than about $10^{-7}$ as in Table II. Obviously, there are large range of parameters satisfying the bounds of Table III, but excluded by the bounds of Table II. Thus $\mu \rightarrow e \gamma$ provides meaningful additional constraints on R-parity violation over the constraints from neutrino masses. It is also true that large range of parameters satisfying the bounds of Table II can be excluded by the bounds of Table III. All bounds of Table I would be trivially satisfied for small $\tan \beta$ if the bounds of Table III are imposed. We still note that if $\tan \beta$ is greater than 10 , some bounds of Table I provide meaningful additional constraints on R-parity violation over the bounds from neutrino masses. Furthermore, if a light sterile neutrino is introduced, it may not be necessary that all of the three weak doublet neutrinos have masses smaller than $1 \mathrm{eV}$ to explain the neutrino oscillation data, which would allow some of the bounds of Table III 
relaxed.

To conclude, we have derived strong bounds on certain products of the bilinear and trilinear R-parity violating couplings from flavor changing decays of the neutral kaon, $\mu^{-}$ $e$ conversion in nuclei, and also $\mu \rightarrow e \gamma$. We also discussed the constraints on R-parity violation from neutrino masses under the assumption that existing neutrino data imply that all the neutrino masses are smaller than $1 \mathrm{eV}$. Wide range of parameter space satisfying the constraints from neutrino masses can be excluded by the bounds from flavor changing processes, and vice versa, thus these two sets of constraints are complementary to each other.

\section{Acknowledgement}

This work is supported by Seoam Foundation (K.C.) and by the KOSEF grant No.19992-111-002-5 and BK21 project of the Ministry of Education (K. C. and K. H.) 


\section{REFERENCES}

[1] For reviews, see for instance H. Dreiner, hep-ph/9707435; G. Bhattacharyya, hepph/9709395.

[2] D. Choudhury and P. Roy, Phys. Lett. B378, 153 (1996).

[3] K. Agashe and M. Graesser, Phys. Rev. D54, 4445 (1996).

[4] K. Huitu, J. Maalampi, M. Raidal and A. Santamaria, Phys. Lett. B430, 355 (1998);

J.E. Kim, P. Ko and D.-G. Lee, Phys. Rev. D56, 100 (1997).

[5] A. Faessler, T.S. Kosmas, S. Kovalenko and J.D. Vergados, hep-ph/9904335.

[6] B. de Carlos and P.L. White, Phys. Rev. D54, 3427 (1996); M. Chaichian and K. Huitu, Phys. Lett. B384, 157 (1996).

[7] K. Choi, E.J. Chun and K. Hwang, hep-ph/0004101.

[8] Y.-Y. Keum, O.C.W. Kong, hep-ph/0004110.

[9] L. Hall and M. Suzuki, Nucl. Phys. B231, 419 (1984).

[10] O. Haug et al., Nucl. Phys. B565, 38 (2000).

[11] A. Abada and M. Losada, hep-ph/9908352.

[12] E.J. Chun and J.S. Lee, Phys. Rev. D60, 075006 (1999).

[13] The Super-Kamiokande Collaboration, Y. Fukuda, et al., Phys. Rev. Lett. 81, 1562 (1998).

[14] M. Bisset, O.C.W. Kong, C. Macesanu and L.H. Orr, Phys. Lett. B430, 274 (1998); Y. Grossman and H.E. Haber, Phys. Rev. D59, 093008 (1999).

[15] T. Banks, Y. Grossman, E. Nardi and Y. Nir, Phys. Rev. D52, 5319 (1995); H. -P. Nilles and N. Polonsky, Nucl. Phys. B484, 33 (1997); S. Davidson and J. Ellis, Phys. Lett. B390, 210 (1997); Phys. Rev. D56, 4182 (1997); S. Davidson, Phys. Lett. B439, 
63 (1998); J. Ferrandis, Phys. Rev. D60, 095012 (1999); S. Davidson, M. Losada and N. Rius, hep-ph/9911317; Y. Grossman and H. E. Haber, preprint in preparation.

[16] A.S. Joshipura and M. Nowakowski, Phys. Rev. D51, 2421 (1995); M. Nowakowski and A. Pilaftsis, Nucl. Phys. B461, 19 (1996).

[17] Particle Data Group, Euro. Phys. J. C1, 1 (1998).

[18] K. Choi, E.J. Chun and K. Hwang, Phys. Rev. D60, 031301 (1999); D. E. Kaplan and A. E. Nelson, JHEP 0001, 033 (2000).

[19] S. Davidson and M. Losada, hep-ph/0005080; See for the full descprition, E.J. Chun and S.K. Kang, Phys. Rev. D61, 075012 (2000). 


\section{TABLES}

\begin{tabular}{|c|c|c|c|}
\hline \hline process & coupling & exchanged scalar mass & upper bound \\
\hline$K_{L} \rightarrow \mu \bar{\mu}$ [2] : & $\lambda_{221}^{\prime} \epsilon_{2} / c_{\beta}$ & $m_{\tilde{u}_{2}}^{2}$ & $5.7 \times 10^{-4} x_{s}^{2}$ \\
& $\lambda_{212}^{\prime} \epsilon_{2} / c_{\beta}$ & $m_{\tilde{\nu}_{2}}^{2}$ & $6.8 \times 10^{-4} x_{s}^{2}$ \\
\hline$K_{L} \rightarrow e \bar{\mu}, \mu \bar{e}$ [2]: & $\lambda_{121}^{\prime} \epsilon_{2} / c_{\beta}, \lambda_{221}^{\prime} \epsilon_{1} / c_{\beta}$ & $m_{\tilde{u}_{2}}^{2}$ & $3.4 \times 10^{-5} x_{s}^{2}$ \\
& $\lambda_{112}^{\prime} \epsilon_{2} c_{\beta}$ & $m_{\tilde{u}_{1}}^{2}, m_{\tilde{\nu}_{1}}^{2}$ & $3.1 \times 10^{-5} x_{s}^{2} x_{g}^{2}$ \\
& $\lambda_{221,212}^{\prime} \epsilon_{1} / c_{\beta}$ & $m_{\tilde{\nu}_{2}}^{2}$ & $4.1 \times 10^{-5} x_{s}^{2}$ \\
\hline$\mu+T i \rightarrow e+T i[5]:$ & $\lambda_{111}^{\prime} \epsilon_{2} c_{\beta}$ & $m_{\tilde{\nu}_{1}}^{2}, m_{\tilde{u}_{1}}^{2}$ & $1.1 \times 10^{-5} x_{s}^{2} x_{g}^{2}$ \\
& $\lambda_{122}^{\prime} \epsilon_{2} / c_{\beta}$ & $m_{\tilde{u}_{2}}^{2}$ & $4.9 \times 10^{-4} x_{s}^{2}$ \\
& $\lambda_{211}^{\prime} \epsilon_{1} / c_{\beta}$ & $m_{\tilde{\nu}_{2}}^{2}$ & $7.4 \times 10^{-6} x_{s}^{2}$ \\
& $\lambda_{222}^{\prime} \epsilon_{1} / c_{\beta}$ & $m_{\tilde{\nu}_{2}}^{2}$ & $1.4 \times 10^{-5} x_{s}^{2}$ \\
\hline$K^{+} \rightarrow \pi^{+} \nu \bar{\nu}[3,2]:$ & $\lambda_{i 12, i 21}^{\prime} \epsilon_{l} c_{\beta}$ & $m_{\tilde{d}_{1,2}}^{2}$ & $1.2 \times 10^{-4} x_{s}^{2} x_{g}$ \\
\hline \hline
\end{tabular}

TABLE I. Bounds on the products of trilinear and bilinear couplings from various flavor changing processes induced by tree diagrams. Here $x_{s} \equiv \frac{m_{\tilde{f}}}{100 \mathrm{GeV}}, x_{g} \equiv \frac{M_{1 / 2}}{100 \mathrm{GeV}}$ and $c_{\beta}=\cos \beta$. 


\begin{tabular}{|c|c|}
\hline \hline coupling & upper bound \\
\hline$\lambda_{1 j 2} \epsilon_{j}^{*} c_{\beta}$ & $1.5 \times 10^{-7} x_{s} x_{g}$ \\
$\lambda_{2 j 1} \epsilon_{j}^{*} c_{\beta}$ & $1.5 \times 10^{-7} x_{s} x_{g}$ \\
$\delta_{1 i}^{L L} \lambda_{i j 2} \epsilon_{j}^{*} c_{\beta}$ & $1.5 \times 10^{-7} x_{s} x_{g}$ \\
$\delta_{2 i}^{L L} \lambda_{i j 1} \epsilon_{j}^{*} c_{\beta}$ & $1.5 \times 10^{-7} x_{s} x_{g}$ \\
$\delta_{1 k}^{R R} \lambda_{2 j k} \epsilon_{j}^{*} c_{\beta}$ & $1.0 \times 10^{-6} x_{s} x_{g}$ \\
$\delta_{2 k}^{R R} \lambda_{1 j k} \epsilon_{j}^{*} c_{\beta}$ & $1.0 \times 10^{-6} x_{s} x_{g}$ \\
$\lambda_{1 j 2} \epsilon_{j}^{*} \frac{\mu v_{2}}{\tilde{m}^{2}}$ & $5.5 \times 10^{-6} x_{s}^{2} x_{g}^{2}$ \\
$\lambda_{2 j 1} \epsilon_{j}^{*} \frac{\mu v_{2}}{\tilde{m}^{2}}$ & $5.5 \times 10^{-6} x_{s}^{2} x_{g}^{2}$ \\
\hline \hline
\end{tabular}

TABLE II. Bounds on the products of trilinear and bilinear couplings from $\mu \rightarrow e \gamma$. Here $x_{s} \equiv \frac{m_{\tilde{f}}}{100 \mathrm{GeV}}, x_{g} \equiv \frac{M_{1 / 2}}{100 \mathrm{GeV}}, c_{\beta}=\cos \beta$, and $\delta_{i j}^{A B}=\Delta_{i j}^{A B} / m_{\tilde{l}_{i}} m_{\tilde{l}_{j}}$ for the off-diagonal elements $\Delta_{i j}^{A B}$ of the slepton mass-squared matrix with $A, B=L, R$ denoting the chirality of sleptons. In all these bounds, $c_{\beta}$ can be replaced by $\frac{M_{W}}{M_{1 / 2}}$. 


\begin{tabular}{|c|c|}
\hline \hline coupling & upper bound \\
\hline$\epsilon_{i}$ & $3 \times 10^{-6} x_{s}^{1 / 2} / c_{\beta}$ \\
$\lambda_{i k l} \lambda_{j l k} y_{k}^{e} y_{l}^{e}$ & $2 \times 10^{-6} x_{s} / t_{\beta}$ \\
$\lambda_{i k l}^{\prime} \lambda_{j l k}^{\prime} y_{k}^{d} y_{l}^{d}$ & $10^{-7} x_{s} / t_{\beta}$ \\
$\epsilon_{i} \lambda_{j k k} y_{k}^{e}$ & $10^{-7} x_{s} / c_{\beta}$ \\
$\epsilon_{i} \lambda_{j k k}^{\prime} y_{k}^{d}$ & $2 \times 10^{-8} x_{s} / c_{\beta}$ \\
$\epsilon_{k} \lambda_{i j k} y_{k}^{e}\left[\left(y_{i}^{e}\right)^{2}-\left(y_{j}^{e}\right)^{2}\right]$ & $5 \times 10^{-4} x_{s} / t_{\beta}$ \\
$\epsilon_{i} \lambda_{j k k}\left(y_{k}^{e}\right)^{3}$ & $5 \times 10^{-4} x_{s}^{3}$ \\
$\epsilon_{i} \lambda_{j k k}^{\prime}\left(y_{k}^{d}\right)^{3}$ & $10^{-5} x_{s}^{3}$ \\
\hline \hline
\end{tabular}

TABLE III. Constraints on R-parity violation from the condition that all elements of the $3 \times 3$ neutrino mass matrix are smaller than $1 \mathrm{eV}$, which would be necessary to account for the neutrino oscillation data. Here $y_{i}^{e} \equiv m_{i}^{e} / m_{\tau}, y_{i}^{d} \equiv m_{i}^{d} / m_{b}$ and $c_{\beta}=\cos \beta, t_{\beta}=\tan \beta$, while $x_{s}=M_{S U S Y} / 100 \mathrm{GeV}$ for the SUSY mass scales $M_{S U S Y}=\left\{M_{1 / 2}, m_{\tilde{f}}, \mu, A^{d, e}\right\}$. 


\section{FIGURES}

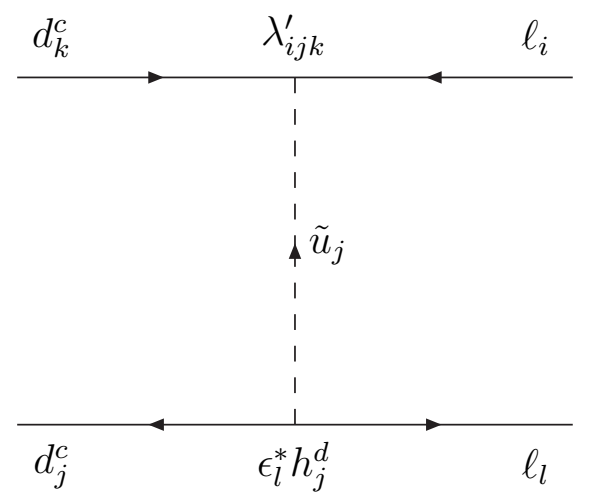

(a)

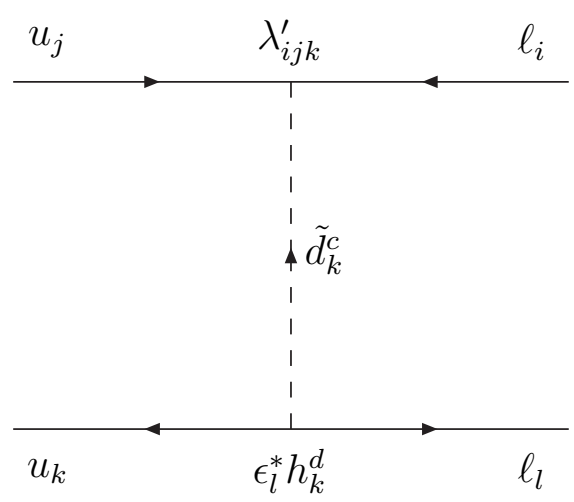

(c)

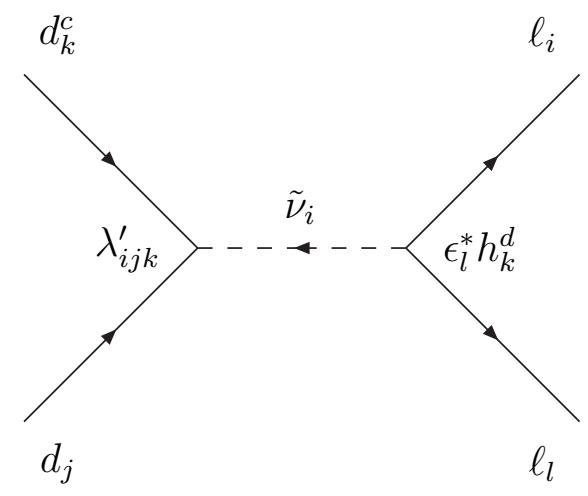

(b)

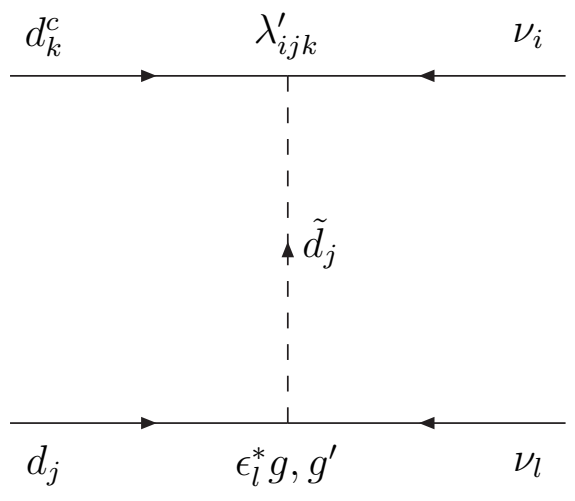

(d)

FIG. 1. Important diagrams for $K_{L} \rightarrow \mu \bar{\mu}, K_{L} \rightarrow e \bar{\mu}, \mu \bar{e}$ (a,b), $\mu+T_{i} \rightarrow e+T_{i}$ (a,b,c) and $K^{+} \rightarrow \pi^{+} \nu \bar{\nu}(\mathrm{d})$ 

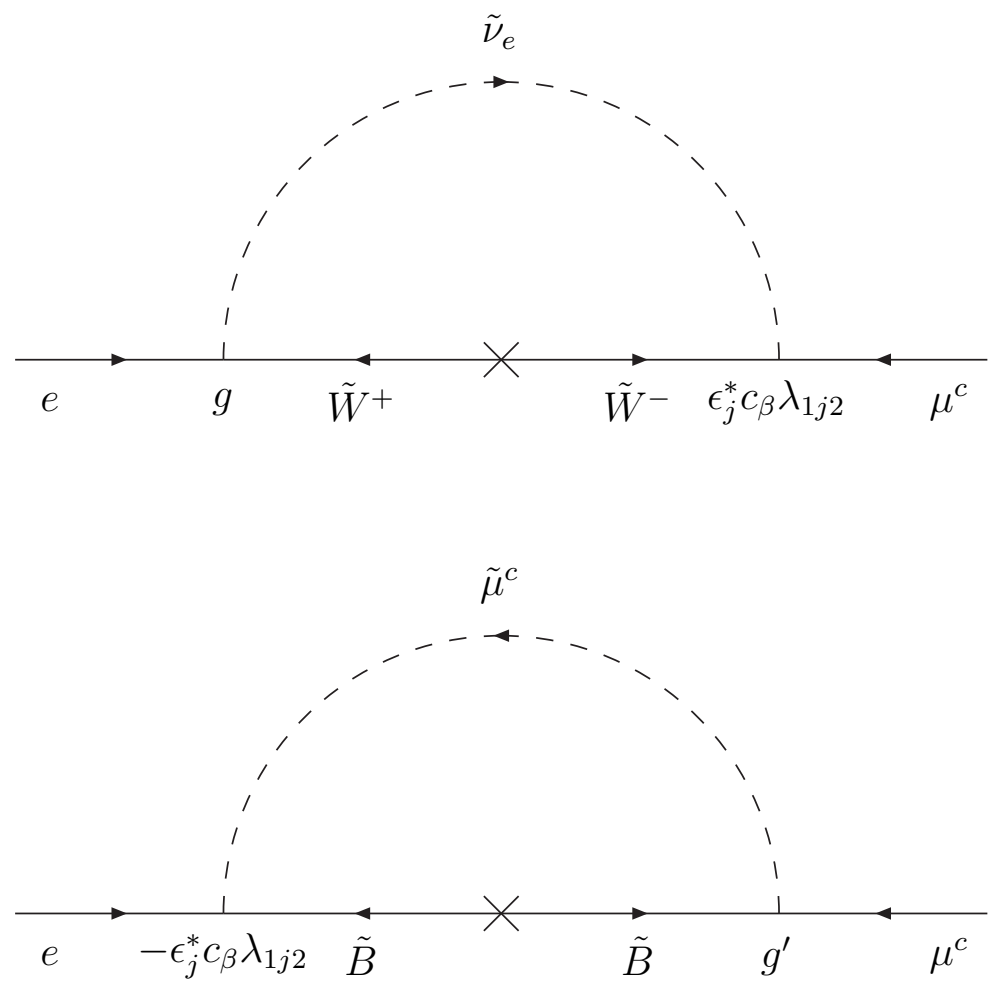

(a)

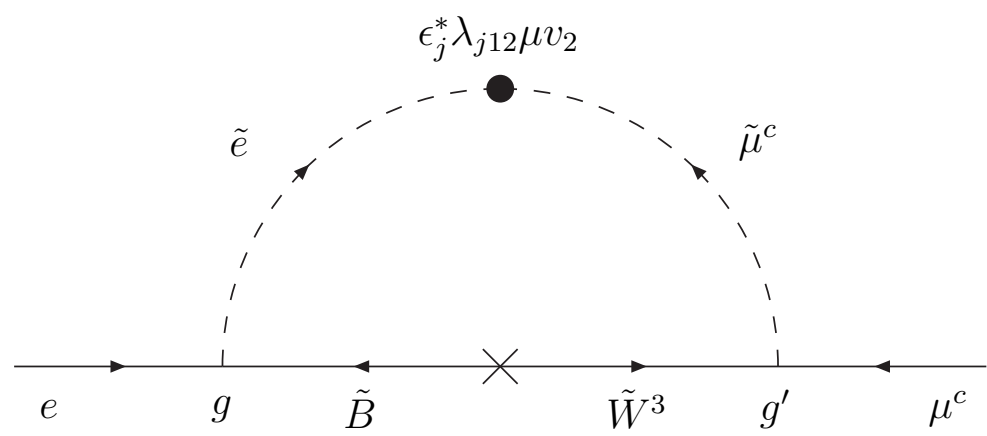

(b)

FIG. 2. Important diagrams for the dipole moment $\mathcal{B}_{R}$ of $\mu \rightarrow e \gamma$ decay. Here, the cross denotes the gaugino mass insertion and the blop denotes the scalar mass mixing. Diagrams for $\mathcal{B}_{L}$ can be obtained by changing $e$ and $\mu$ each other and also $\lambda_{1 j 2}$ by $\lambda_{2 j 1}$. 